\title{
Ethanol Production from Nondetoxified Dilute-Acid Lignocellulosic Hydrolysate by Cocultures of Saccharomyces cerevisiae Y5 and Pichia stipitis CBS6054
}

\author{
Ping Wan, Dongmei Zhai, Zhen Wang, Xiushan Yang, and Shen Tian \\ College of Life Science, Capital Normal University, Beijing 100048, China \\ Correspondence should be addressed to Shen Tian, cnu_tianshen@yahoo.com.cn
}

Received 16 April 2012; Revised 15 May 2012; Accepted 22 May 2012

Academic Editor: Hongjuan Liu

Copyright ( $) 2012$ Ping Wan et al. This is an open access article distributed under the Creative Commons Attribution License, which permits unrestricted use, distribution, and reproduction in any medium, provided the original work is properly cited.

\begin{abstract}
Saccharomyces cerevisiae Y5 (CGMCC no. 2660) and Issatchenkia orientalis Y4 (CGMCC no. 2159) were combined individually with Pichia stipitis CBS6054 to establish the cocultures of Y5 + CBS6054 and Y4 + CBS6054. The coculture Y5 + CBS6054 effectively metabolized furfural and HMF and converted xylose and glucose mixture to ethanol with ethanol concentration of $16.6 \mathrm{~g} / \mathrm{L}$ and ethanol yield of $0.46 \mathrm{~g}$ ethanol/g sugar, corresponding to $91.2 \%$ of the maximal theoretical value in synthetic medium. Accordingly, the nondetoxified dilute-acid hydrolysate was used to produce ethanol by co-culture Y5 + CBS6054. The co-culture consumed glucose along with furfural and HMF completely in $12 \mathrm{~h}$, and all xylose within $96 \mathrm{~h}$, resulting in a final ethanol concentration of $27.4 \mathrm{~g} / \mathrm{L}$ and ethanol yield of $0.43 \mathrm{~g}$ ethanol $/ \mathrm{g}$ sugar, corresponding to $85.1 \%$ of the maximal theoretical value. The results indicated that the co-culture of Y5 + CBS6054 was a satisfying combination for ethanol production from non-detoxified diluteacid lignocellulosic hydrolysates. This co-culture showed a promising prospect for industrial application.
\end{abstract}

\section{Introduction}

Cellulosic ethanol has been widely regarded as a promising alternative liquid fuel due to its projected positive attributes in terms of economic, environmental, and social sustainability [1-3]. The ability to generate and convert fermentable sugars from lignocellulosic materials to ethanol is the central technological challenge $[4,5]$. The fermentability of a hydrolysate is strongly dependent on the feedstock, the pretreatment method, and the strain selected. Most biomass feedstock contains a significant amount of xylan that is converted to xylose through hydrolysis. Most biomass pretreatment methods, applied to remove barriers to enzymatic cellulose saccharification, produce fermentation inhibitors. Therefore the selected strain needs to be capable of fermenting xylose and glucose with good toleration of inhibitors.

Dilute-acid pretreatment is one of the most promising pretreatment methods for sugar production from lignocelluloses and has been widely studied [6]. However it produces fermentation inhibitory compounds, such as furfural and HMF, the most investigated and the most highly toxic inhibitors. A furfural concentration as high as $1.5 \mathrm{~g} \mathrm{~L}^{-1}$ could interfere respiration and growth of microorganisms, which resulted in the reduction of ethanol yield and productivity by $90.4 \%$ and $85.1 \%$, respectively [7]. The inhibitive effect of HMF is similar to that of furfural, causing an extended lag phase during the growth of microorganism cells. Pichia stipitis growth was reduced by $43 \%, 70 \%$, and $100 \%$ when $\mathrm{HMF}$ concentration in the medium was $0.5,0.75$, and $1.5 \mathrm{~g} \mathrm{~L}^{-1}$, respectively. Additionally, there was a synergistic effect when the two toxic compounds were combined with several other compounds present in the dilute-acid pretreatment hydrolysate $[8,9]$. Reducing the concentrations of these inhibitors in a hydrolysate through removal or dilution can improve the fermentability of the hydrolysate. Several detoxification methods have been developed and proved to be effective [10]. However, most detoxification processes result in sugar reductions and thereby increase cost.

Only a few native yeast strains have the ability to grow in hydrolysates with inhibitors, such as S. cerevisiae TMB 3400, TMB 3006 and Coniochaeta ligniaria NRRL30616, which can slowly convert furfural and HMF to less toxic compounds 
and ferment glucose to ethanol [11]. However, almost none of these strains is able to convert xylose to ethanol. Metabolic engineering can be used for the development of recombinant S. cerevisiae strains to ferment xylose. However, the ability of individual recombinant yeast to tolerate the inhibitors present in the hydrolysates is also an important factor for effective production of cellulosic ethanol and yet needs to be improved [12-14].

An alternative approach is cocultivation of two microorganisms in a single process. A co-culture of Zymomonas mobilis and Pachysolen tannophilus was used to ferment a simulating hydrolysate without inhibitors [15]. We used cocultures of $S$. cerevisiae + Pachysolen tannmphilus and $S$. cerevisiae + Escherichia coli to ferment a detoxified softwood hydrolysate in a previous study [9]. We achieved an ethanol yield of $0.49 \mathrm{~g}$ ethanol $/ \mathrm{g}$ sugars after $72 \mathrm{~h}$, corresponding to $96.1 \%$ of the maximum theoretical ethanol yield. We boiled the hydrolysate for detoxification and the co-culture was adapted before ethanol fermentation.

Few studies reported cofermentation from nondetoxified lignocellulosic hydrolysates. The objective of the present study is to demonstrate the feasibility of cofermentation nondetoxified dilute acid pretreated lignocelluloses hydrolysates using two robust strains, S. cerevisiae Y5 and Issatchenkia orientalis Y4, recently developed in our lab [16]. The two strains Y5 and Y4 have shown high efficiency in converting glucose to ethanol while metabolized furfural and 5-HMF at high concentrations. When the two strains were cultivated in the medium supplemented with $1.0 \mathrm{~g} / \mathrm{L}$ of combined furfural and HMF, the cell growth was largely unaffected. It only produced a $12 \mathrm{~h}$ lag phase in cell growth when the combined inhibitors concentration was increased to $3 \mathrm{~g} / \mathrm{L}$. It is therefore conceivable to coculture S. cerevisiae Y5 and Issatchenkia orientalis Y4, respectively, with $P$. stipitis CBS6054, a xylose fermenting strain, for efficient cofermentation of glucose and xylose in nondetoxified lignocelluloses hydrolysates.

\section{Material and Methods}

2.1. Yeast Strains and Inoculum Preparation. P. stipitis CBS6054 was obtained from ATCC, S. cerevisiae Y5 (CGMCC 2660) and Issatchenkia orientalis Y4 (CGMCC 2159) were preserved in China General Microbiological Culture Collection Center (CGMCC). Y5 was isolated from soil samples of an ethanol industry. Y4 was isolated from soil samples of a furfural industry in glucose medium with furfural added. All strains were maintained on YPD medium: $10 \mathrm{~g} \mathrm{~L}^{-1}$ yeast extract, $20 \mathrm{~g} \mathrm{~L}^{-1}$ peptone, $20 \mathrm{~g} \mathrm{~L}^{-1}$ glucose, and $20 \mathrm{~g} \mathrm{~L}^{-1}$ agar at $4^{\circ} \mathrm{C}$. The inoculants were prepared in $250 \mathrm{~mL}$ plastic-plugged shake flasks with $100 \mathrm{~mL} \mathrm{YP} \mathrm{medium} \mathrm{at} 30^{\circ} \mathrm{C}$ with shaking at $150 \mathrm{rpm}$ overnight. The cells were collected by centrifugation at $5000 \mathrm{rpm}$ for $5 \mathrm{~min}$ at $4^{\circ} \mathrm{C}$ and washed with sterile water twice.

2.2. Medium and Hydrolysate. Glucose medium contained $24 \mathrm{~g} \mathrm{~L}^{-1}$ glucose, $10 \mathrm{~g} \mathrm{~L}^{-1}$ yeast extract, and $20 \mathrm{gL}^{-1}$ peptone. Xylose medium contained $13 \mathrm{gL}^{-1}$ xylose, $10 \mathrm{gL}^{-1}$ yeast extract, and $20 \mathrm{~g} \mathrm{~L}^{-1}$ peptone. Sugar mixture medium was composed of $23.5 \mathrm{~g} \mathrm{~L}^{-1}$ glucose, $13 \mathrm{~g} \mathrm{~L}^{-1}$ xylose, $10 \mathrm{~g} \mathrm{~L}^{-1}$ yeast extract, and $20 \mathrm{~g} \mathrm{~L}^{-1}$ peptone.

The concentrations of furfural and HMF added to the medium were $1.37 \mathrm{~g} \mathrm{~L}^{-1}$ and $0.47 \mathrm{~g} \mathrm{~L}^{-1}$, respectively, equal to the amounts found in the dilute acid pretreatment hydrolysate used in this study. Therefore, comparisons of ethanol production can be made between medium and real pretreatment hydrolysate. The concentrations of 1.37 and $0.47 \mathrm{~g} \mathrm{~L}^{-1}$ are also very close to the levels of 1.5 and $0.5 \mathrm{~g} \mathrm{~L}^{-1}$ for furfural and HMF, respectively, at which significant inhibitions were observed as discussed previously [7, 8]. All of the medium were autoclaved at $115^{\circ} \mathrm{C}$ for 25 minutes.

Hydrolysate from dilute-acid pretreatment of softwood was provided by Department of Energy Chemical Engineering, East China University of Science and Technology, Shanghai, China. Softwood chips were immersed in a $2 \%$ $\mathrm{HCl}$ and $0.5 \% \mathrm{FeCl}_{2}(\mathrm{v} / \mathrm{v})$ solution at $170^{\circ} \mathrm{C}$ for $30 \mathrm{~min}$. The liquid stream, hydrolysate, from the acid hydrolysis was recovered through filtration. The hydrolysate was neutralized with $\mathrm{Ca}(\mathrm{OH})_{2}$ to $\mathrm{pH} 5.0$ prior to fermentation. Yeast extract $\left(10 \mathrm{~g} \mathrm{~L}^{-1}\right)$ and peptone $\left(20 \mathrm{gL}^{-1}\right)$ were added to the hydrolysate and autoclaved at $115^{\circ} \mathrm{C}$ for $25 \mathrm{~min}$. The sterilized hydrolysate medium contained $41.87 \mathrm{~g} \mathrm{~L}^{-1}$ glucose, $22.58 \mathrm{~g} \mathrm{~L}^{-1}$ xylose, $1.37 \mathrm{~g} \mathrm{~L}^{-1}$ furfural, $0.47 \mathrm{~g} \mathrm{~L}^{-1} 5-\mathrm{HMF}$.

2.3. Fermentation. Batch fermentation experiments were performed at $30^{\circ} \mathrm{C}, 80 \mathrm{rpm}$ in $250 \mathrm{~mL}$ flask containing $100 \mathrm{~mL}$ fermentation medium (glucose medium, xylose medium, and lignocellulosic hydrolysate). The initial cell concentrations (dry cell weight) of Y5, Y4, and CBS6054 were $3.3 \mathrm{~g} \mathrm{~L}^{-1}, 2.7 \mathrm{~g} \mathrm{~L}^{-1}$, and $3.0 \mathrm{~g} \mathrm{~L}^{-1}$, respectively.

2.4. Measurement of the Composition of Coculture. The cell concentrations of the co-culture were determined by counting cells in a given sample volume under a microscope (Olympus B-201). The dead cells were differentiated using methylene blue dye which revealed that most of the cells were alive. The cell biomass concentrations were calculated using the measured cell number concentrations and a conversion factor of $1 \times 10^{11}$ and $3 \times 10^{6} \mathrm{~g}^{-1}$ dry strain mass for $P$. stipitis and $S$. cerevisiae, respectively [17].

2.5. Analytical Methods. Ethanol analysis in the cellulosic substrate fermentation broth was carried out using a gas chromatograph (GC, model 7890, Agilent Technologies, Palo Alto, CA) through direct sample injection using an external standard for calibration. The sample was centrifuged and the supernatant was filtered before injection to the GC column. The chromatograph is equipped with a flame ionization detector and Agilent DB Wax column of $30 \mathrm{~m}$ with an ID $0.32 \mathrm{~mm}$. A universal guard column was used to reduce column contamination. Sugar and inhibitor concentrations were measured using an HPLC equipped with an Econosphere C18 column ( $5 \mathrm{~mm}$ particle size, $250 \mathrm{~mm} \times 4.6 \mathrm{~mm}$, Alltech, Deerfield, IL) and a UV1000 ultraviolet detector (277 nm; Thermo Finnigan, San Jose, CA). Samples were run at ambient temperature and eluted at $0.8 \mathrm{~mL} / \mathrm{min}$ 


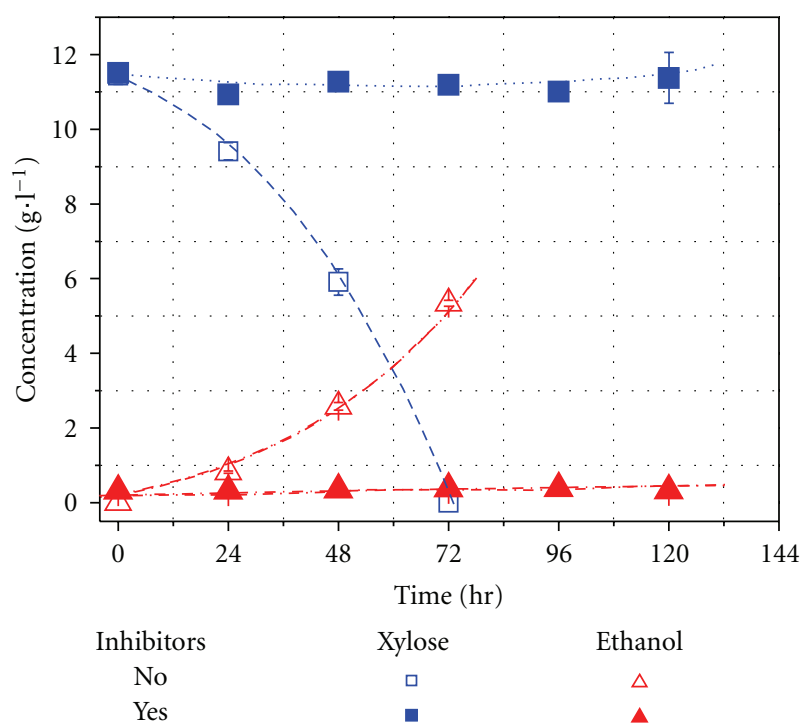

Figure 1: Sugar consumption and ethanol production by CBS6054 in xylose medium without inhibitors and in xylose medium with inhibitors (furfural and 5-HMF concentrations of 1.37 and $0.47 \mathrm{~g} \mathrm{~L}^{-1}$, respectively).

with a linear gradient of $50 \%$ to $100 \%$ acidified methanol (containing $0.25 \%$ acetic acid) run over $15 \mathrm{~min}$.

\section{Results and Discussion}

3.1. Performance of Y5, Y4, and CBS6054 in the Medium Containing Furfural and HMF. In the absence of furfural and HMF in the medium, strains Y5 and Y4 converted all glucose within $12 \mathrm{~h}$ with volumetric ethanol productivity of $0.90 \mathrm{~g} \mathrm{~L}^{-1} \mathrm{~h}^{-1}$ and $0.93 \mathrm{~g} \mathrm{~L}^{-1} \mathrm{~h}^{-1}$, respectively, and strain CBS6054 converted all glucose within $24 \mathrm{~h}$ with volumetric ethanol productivity of $0.47 \mathrm{~g} \mathrm{~L}^{-1} \mathrm{~h}^{-1}$. It was evident that the glucose utilization rate of $\mathrm{Y} 5$ and $\mathrm{Y} 4$ was much higher than that of CBS6054. This outcome may be resulted from the higher cell growth rate of Y5 and Y4. The addition of furfural and HMF resulted in a $12 \mathrm{~h}$ lag time of CBS6054, but it had no obvious impact on Y5 and Y4. The volumetric ethanol productivity of Y5, Y4, and CBS6054 in the glucose medium containing furfural and HMF was $0.94 \mathrm{~g} \mathrm{~L}^{-1} \mathrm{~h}^{-1}$, $0.90 \mathrm{~g} \mathrm{~L}^{-1} \mathrm{~h}^{-1}$, and $0.31 \mathrm{~g} \mathrm{~L}^{-1} \mathrm{~h}^{-1}$, respectively. The results indicated that $\mathrm{Y} 5$ and $\mathrm{Y} 4$ are the desirable strains for ethanol fermentation from dilute-acid lignocellulosic hydrolysates; however, both strains are not capable of utilizing xylose.

It is well known that $P$. stipitis can ferment xylose to ethanol [18]. To establish some baseline for the present study, xylose fermentation using CBS6054 was carried out. Xylose, in a medium with an initial concentration of $11.4 \mathrm{~g} \mathrm{~L}^{-1}$, was used up within $72 \mathrm{~h}$. The final ethanol concentration reached $5.3 \mathrm{~g} \mathrm{~L}^{-1}$, equivalent to $93.4 \%$ of the maximum theoretical value, and the volumetric ethanol productivity was $0.074 \mathrm{~g} \mathrm{~L}^{-1} \mathrm{~h}^{-1}$. However, the growth of strain CBS6054 was totally inhibited when $1.37 \mathrm{~g} \mathrm{~L}^{-1}$ furfural and $0.47 \mathrm{~g} \mathrm{~L}^{-1}$

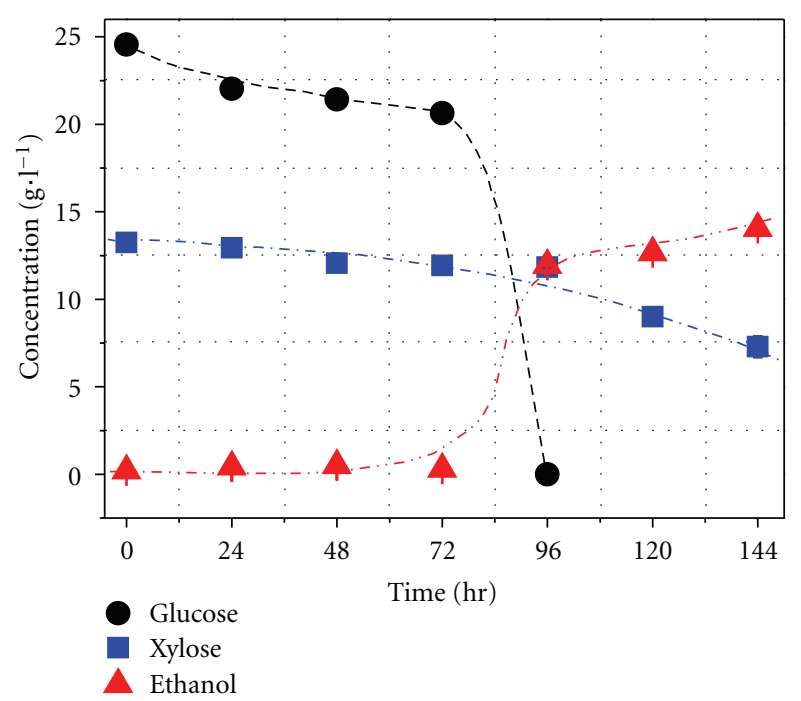

FIGURE 2: Sugar consumption and ethanol production by CBS6054 in mixture medium with furfural and 5-HMF concentrations of 1.37 and $0.47 \mathrm{~g} \mathrm{~L}^{-1}$, respectively.

HMF were added to the medium (Figure 1), similar phenomena were observed by Agbogbo et al. [8].

The consumption of glucose/xylose mixture containing furfural and HMF and ethanol yield by $P$. stipitis CBS6054 are shown in Figure 2. The glucose was completely consumed within $96 \mathrm{~h}$. Xylose metabolization was initiated in $72 \mathrm{~h}$. Xylose was partially converted to ethanol eventually. Compared to the results obtained in a medium without glucose with the same amount of inhibitors (Figure 1), the results shown in Figure 2 suggest that glucose reduced the inhibitive effect on CBS6054.

From the results obtained in inhibitor containing media discussed in this subsection, we hypothesize that the individual strains of Y5 and Y4 could be combined with strain CBS6054 to establish two co-cultures and the co-cultures may exhibit excellent characteristics for fermentation, for example, high glucose and xylose conversion rate, high inhibitor tolerance along with high ethanol yield from diluteacid lignocellulosic hydrolysate without detoxification.

3.2. Performance of Coculture in Medium Containing Furfural and HMF. Co-cultures of Y4 + CBS6054 and Y5 + CBS6054 were investigated in sugar mixture medium containing inhibitors. The consumption of glucose/xylose and the ethanol yield were shown in Figures 3 and 4. The glucose/xylose consumption and ethanol production by coculture Y4 + CBS6054 were shown in Figure 3. Only about $20 \%$ of the xylose was consumed after 144 hours fermentation, suggesting the co-culture was not successful for cofermenting glucose and xylose medium with inhibitors. The reason for this may be that xylose metabolism of strain CBS6054 was interfered with the metabolites of strain Y4.

The fermentation results of co-culture Y5 + CBS6054 were promising; xylose was successfully used up within $144 \mathrm{~h}$ (Figure 4). Both furfural and HMF were completely 


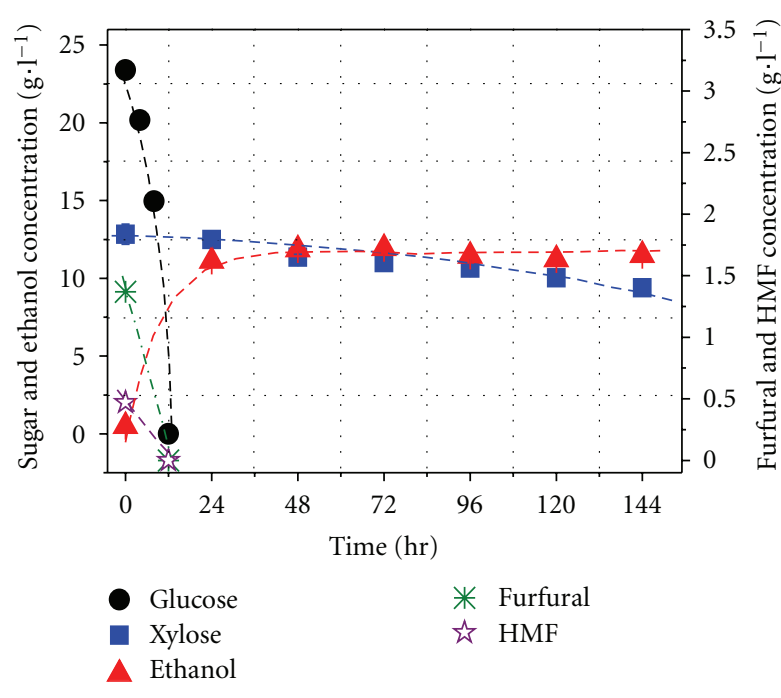

FIGURE 3: Sugar consumption and ethanol production by coculture Y4 + CBS6054 in mixture medium containing inhibitors.

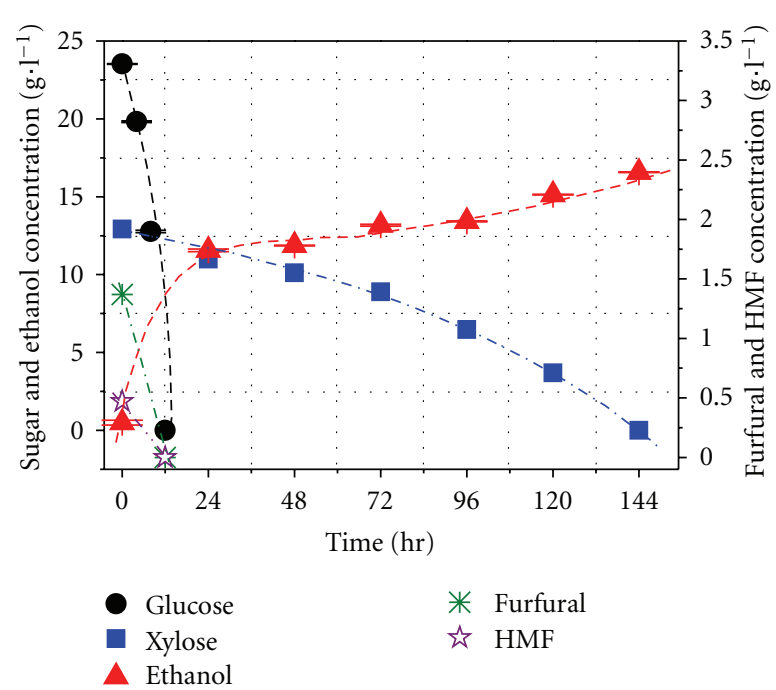

FIGURE 4: Sugar consumption and ethanol production by coculture Y5 + CBS6054 in sugar mixture medium containing inhibitors.

metabolized along with glucose exhausted in $12 \mathrm{~h}$. The final ethanol concentrations in sugar mixture medium without (not shown for clarity) and with inhibitors were $16.6 \mathrm{~g} \mathrm{~L}^{-1}$ and $15.8 \mathrm{~g} \mathrm{~L}^{-1}$, respectively, corresponding to ethanol yields 0.46 and 0.43 ethanol/g sugar, equivalent to $89.2 \%$ and $84.9 \%$ of the maximum theoretical yield, with the volumetric ethanol productivity $0.12 \mathrm{~g} \mathrm{~L}^{-1} \mathrm{~h}^{-1}$ and $0.11 \mathrm{~g} \mathrm{~L}^{-1} \mathrm{~h}^{-1}$, respectively. The results indicate that the inhibitive effect is negligible for the Y5 + CBS6054, suggesting our hypothesis for the Y5 + CBS6054 co-culture is valid.

To demonstrate the effectiveness of the Y5 + CBS6054 co-culture, xylose consumptions and ethanol yields from fermenting the mixture sugar of glucose and xylose containing furfural and 5-HMF by CBS6054 alone and the co-culture CBS6054 + Y5 were compared (Figure 5).

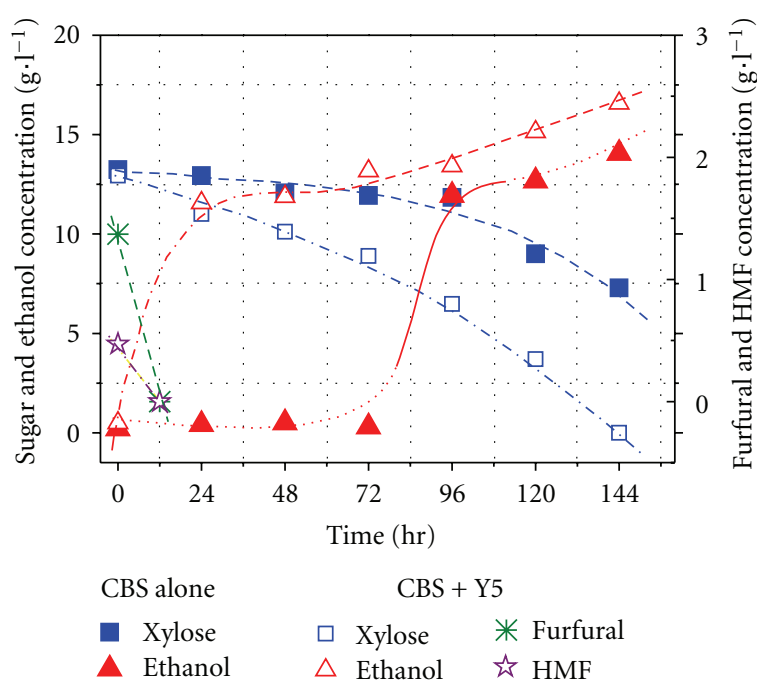

FIGURE 5: Xylose consumption and ethanol production by CBS6054 and Y5 + CBS6054 in mixture medium containing inhibitors.

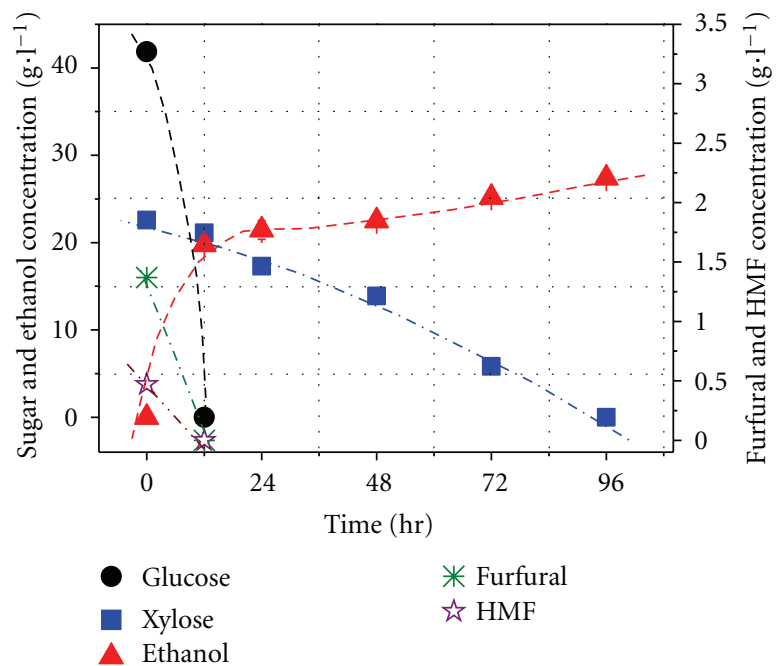

FIGURE 6: Glucose, xylose consumption and ethanol production by co-culture Y5 + CBS6054 in dilute-acid lignocellulosic hydrolysate without detoxification.

The results clearly indicate that xylose metabolism by CBS6054 was improved when cocultured with Y5, suggesting the synergetic effect of co-culture for xylose fermentation. The metabolization of the inhibitors, that is, furfural and HMF by Y5 certainly improves xylose fermentation by CBS6054, which resulted in a final high ethanol yield.

\subsection{Fermentation of Nondetoxified Hydrolysate by Co-Culture} Y5 + CBS6054. Since co-culture Y5 + CBS6054 showed efficient fermentation in a synthetic glucose/xylose medium with inhibitors, further experiments were carried out to investigate the fermentation characteristics of the nondetoxified hydrolysate by co-culture Y5 + CBS6054 (Figure 6). Glucose was used up and furfural and HMF was completely metabolized within $12 \mathrm{~h}$ with agitation speed of $80 \mathrm{rpm}$. 


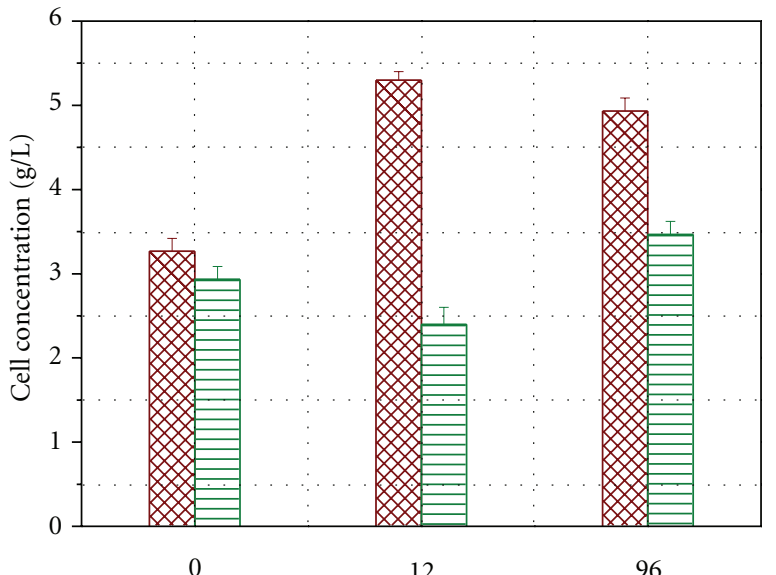

(h)

S. cerevisiae Y5 P. stipitis CBS6054

FIgURE 7: Cell concentration of Y5 and CBS6054 during fermentation in dilute-acid lignocellulosic hydrolysate without detoxification.

Xylose was used up within $96 \mathrm{~h}$. The final ethanol concentration and yield was $27.4 \mathrm{~g} \mathrm{~L}^{-1}$ and $0.43 \mathrm{~g}$ ethanol/g sugar, respectively, corresponding to $85.1 \%$ of the maximal theoretical value with ethanol productivity of $0.29 \mathrm{~g} \mathrm{~h}^{-1} \mathrm{~L}^{-1}$.

The cell biomass of the two yeast strains in co-culture Y5 + CBS6054 was measured during the fermentation process (Figure 7). At 0,12 , and $96 \mathrm{~h}$, the ratio of Y5 to CBS6054 was roughly $1: 1,2: 1$ and $3: 2$, respectively. The results indicated that $\mathrm{Y} 5$ had a high growth rate during the period of glucose consumption $(<12 \mathrm{~h})$. With the degradation of furfural and 5-HMF, CBS6054 began to slowly convert xylose to ethanol without being restrained and CBS6054 cell biomass showed moderate increase $(>12 \mathrm{~h})$. As glucose was consumed, the cell biomass of Y5 was decreased. As a result, the cell mass ratio between Y5 and CBS6054 was declined substantially to about $3: 2$ as fermentation further proceeds to the end ( $96 \mathrm{~h})$.

\section{Conclusions}

To realize the industrial ethanol production from dilute-acid pretreated lignocellulosic hydrolysate, it is essential to obtain the strains that are able to convert glucose and xylose to ethanol as well as metabolize inhibitors in the hydrolysate. There are two approaches which can achieve this objective. One is to find a satisfactory co-culture, and the other is to construct the new strains to convert both glucose and xylose to ethanol.

This study demonstrated an effective co-culture of Y5 + CBS6054 to ferment lignocellulosic hydrolysate. The coculture exhibited some good features: that is, converting glucose and xylose to ethanol, as well as effectively degrading inhibitors in the hydrolysate. The glucose was used up and furfural and 5-HMF were completely metabolized within $12 \mathrm{~h}$; xylose was used up in $96 \mathrm{~h}$ at $80 \mathrm{rpm}$ with ethanol concentration and yield of $27.4 \mathrm{~g} \mathrm{~L}^{-1}$ and $0.43 \mathrm{~g}$ ethanol/g sugar, respectively, corresponding to $85.1 \%$ of the maximal theoretical value with ethanol productivity of $0.29 \mathrm{~g} \mathrm{~h}^{-1} \mathrm{~L}^{-1}$ without detoxification of the hydrolysate. In conclusion, the Y5 + CBS6054 co-culture presents a promising approach for ethanol production from dilute-acid pretreated lignocellulosic hydrolysate. The optimum growth conditions of the yeasts in cocultivation will be studied in the future.

\section{Authors' Contribution}

P. Wan and D. Zhai contributed equally to this work.

\section{Acknowledgments}

The authors acknowledge financial support received from the National Key Technology R\&D Program (no. 2011BAD22B01), the National Natural Science Foundation of China (no. 31100578).

\section{References}

[1] B. H. Hägerdal, K. Karhumaa, C. Fonseca, I. S. Martins, and M. F. G. Grauslund, "Towards industrial pentose-fermenting yeast strains," Applied Microbiology and Biotechnology, vol. 74, no. 5, pp. 937-953, 2007.

[2] S. Katahira, A. Mizuike, H. Fukuda, and A. Kondo, "Ethanol fermentation from lignocellulosic hydrolysate by a recombinant xylose- and cellooligosaccharide-assimilating yeast strain," Applied Microbiology and Biotechnology, vol. 72, no. 6, pp. 1136-1143, 2006.

[3] L. Laopaiboon, P. Thanonkeo, P. Jaisil, and P. Laopaiboon, "Ethanol production from sweet sorghum juice in batch and fed-batch fermentations by Saccharomyces cerevisiae," World Journal of Microbiology and Biotechnology, vol. 23, no. 10, pp. 1497-1501, 2007.

[4] R. P. Chandra, R. Bura, W. E. Mabee, A. Berlin, X. Pan, and J. N. Saddler, "Substrate pretreatment: the key to effective enzymatic hydrolysis of lignocellulosics," Advances in Biochemical Engineering/Biotechnology, vol. 108, pp. 67-93, 2007.

[5] B. S. Dien, M. A. Cotta, and T. W. Jeffries, "Bacteria engineered for fuel ethanol production: current status," Applied Microbiology and Biotechnology, vol. 63, no. 3, pp. 258-266, 2003.

[6] M. Chen, J. Zhao, and L. Xia, "Comparison of four different chemical pretreatments of corn stover for enhancing enzymatic digestibility," Biomass and Bioenergy, vol. 33, no. 10, pp. 1381-1385, 2009.

[7] F. K. Agbogbo and K. S. Wenger, "Production of ethanol from corn stover hemicellulose hydrolyzate using Pichia stipitis," Journal of Industrial Microbiology and Biotechnology, vol. 34, no. 11, pp. 723-727, 2007.

[8] F. K. Agbogbo and K. S. Wenger, "Effect of pretreatment chemicals on xylose fermentation by Pichia stipitis," Biotechnology Letters, vol. 28, no. 24, pp. 2065-2069, 2006.

[9] M. Qian, S. Tian, X. Li, J. Zhang, Y. Pan, and X. Yang, "Ethanol production from dilute-acid softwood hydrolysate by co-culture," Applied Biochemistry and Biotechnology, vol. 134, no. 3, pp. 273-283, 2006.

[10] R. E. Berson, S. John, S. N. Kamer, and R. H. Thomas, "Detoxification of actual pretreated corn stover hydrolysate using activated carbon powder," Applied Biochemistry and Biotechnology A, vol. 124, no. 1-3, pp. 923-934, 2005. 
[11] N. N. Nichols, B. S. Dien, G. M. Guisado, and M. J. López, "Bioabatement to remove inhibitors from biomass-derived sugar hydrolysates," Applied Biochemistry and Biotechnology A, vol. 121, no. 1-3, pp. 379-390, 2005.

[12] J. R. M. Almeida, A. Röder, T. Modig, B. Laadan, G. Lidén, and M. F. G. Grauslund, "NADH- vs NADPH-coupled reduction of 5-hydroxymethyl furfural (HMF) and its implications on product distribution in Saccharomyces cerevisiae," Applied Microbiology and Biotechnology, vol. 78, no. 6, pp. 939-945, 2008.

[13] A. Saloheimo, J. Rauta, O. V. Stasyk, A. A. Sibirny, M. Penttilä, and L. Ruohonen, "Xylose transport studies with xyloseutilizing Saccharomyces cerevisiae strains expressing heterologous and homologous permeases," Applied Microbiology and Biotechnology, vol. 74, no. 5, pp. 1041-1052, 2007.

[14] M. Sedlak and N. W. Ho, "Production of ethanol from cellulosic biomass hydrolysates using genetically engineered Saccharomyces yeast capable of cofermenting glucose and xylose," Applied Biochemistry and Biotechnology, vol. 113-116, pp. 403-416, 2004.

[15] N. Fu and P. Peiris, "Co-fermentation of a mixture of glucose and xylose to ethanol by Zymomonas mobilis and Pachysolen tannophilus," World Journal of Microbiology and Biotechnology, vol. 24, no. 7, pp. 1091-1097, 2008.

[16] S. Tian, G. Zhou, F. Yan, Y. Yu, and X. Yang, "Yeast strains for ethanol production from lignocellulosic hydrolysates during in situ detoxification," Biotechnology Advances, vol. 27, no. 5, pp. 656-660, 2009.

[17] I. de Bari, D. Cuna, F. Nanna, and G. Braccio, "Ethanol production in immobilized-cell bioreactors from mixed sugar syrups and enzymatic hydrolysates of steam-exploded biomass," Applied Biochemistry and Biotechnology A, vol. 114, no. 1-3, pp. 539-557, 2004.

[18] T. W. Jeffries, I. V. Grigoriev, J. Grimwood et al., "Genomesequence of the lignocellulose-bioconverting and xylosefermenting yeast Pichia stipitis," Nature Biotechnology, vol. 25, no. 3, pp. 319-326, 2007. 

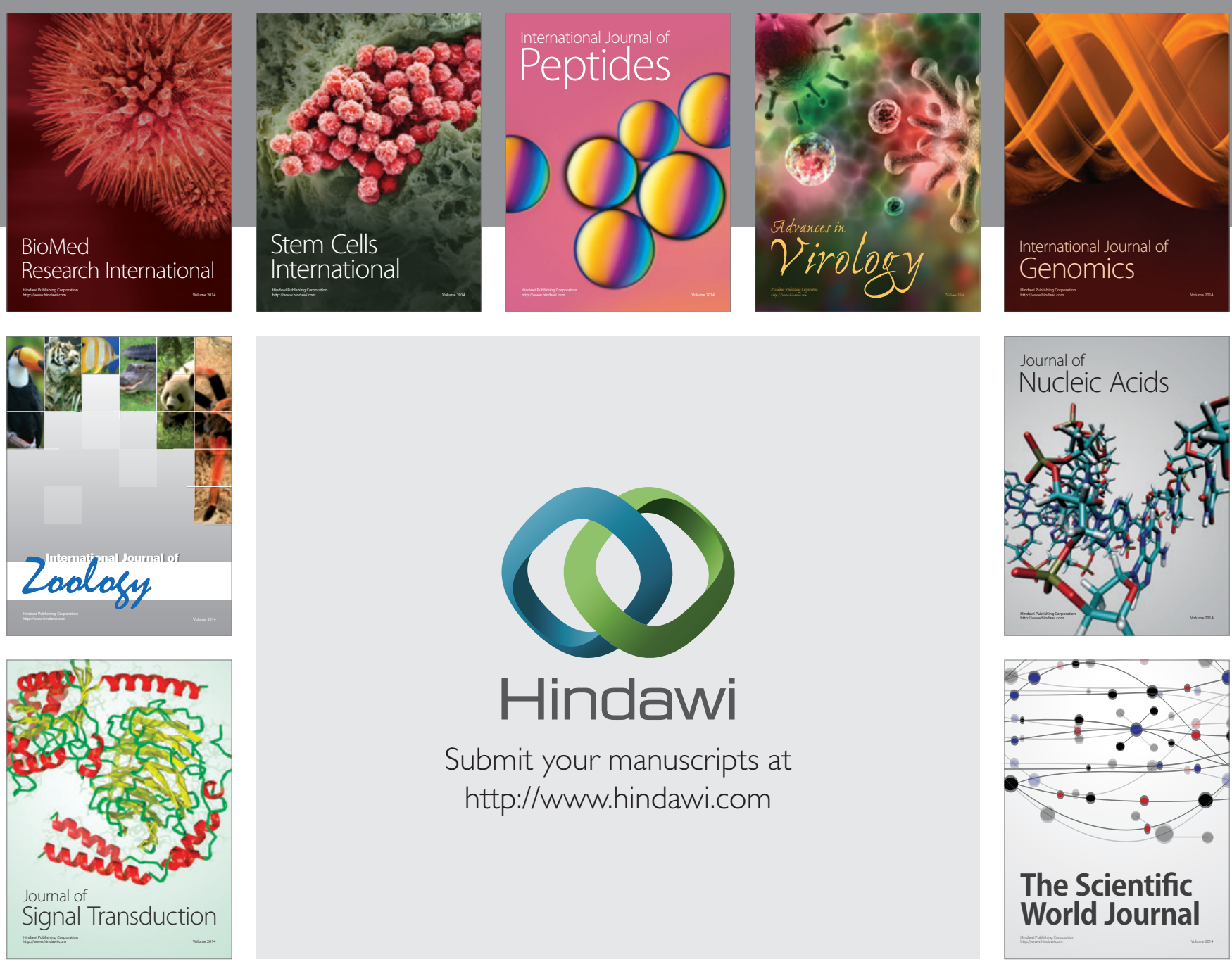

Submit your manuscripts at

http://www.hindawi.com
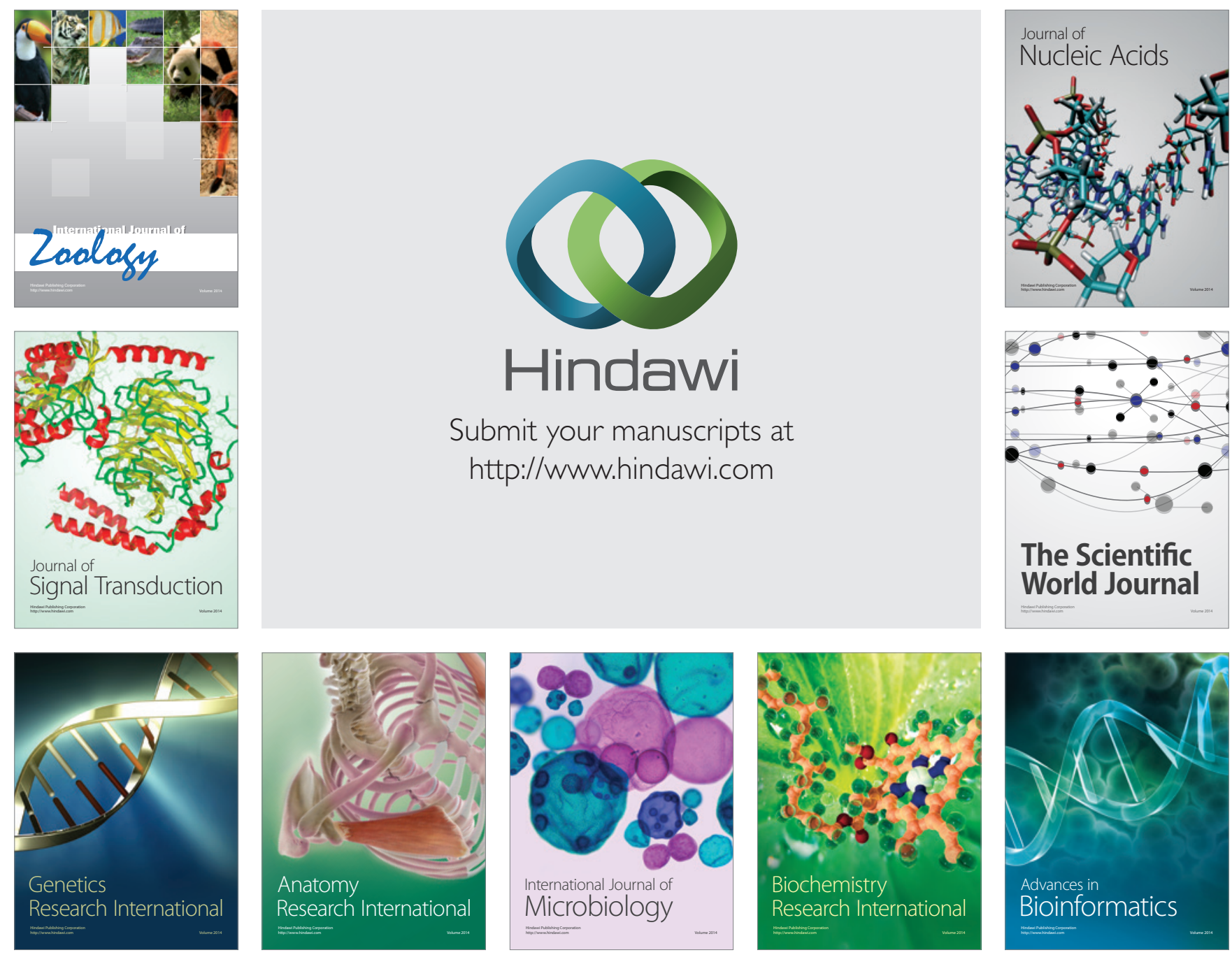

The Scientific World Journal
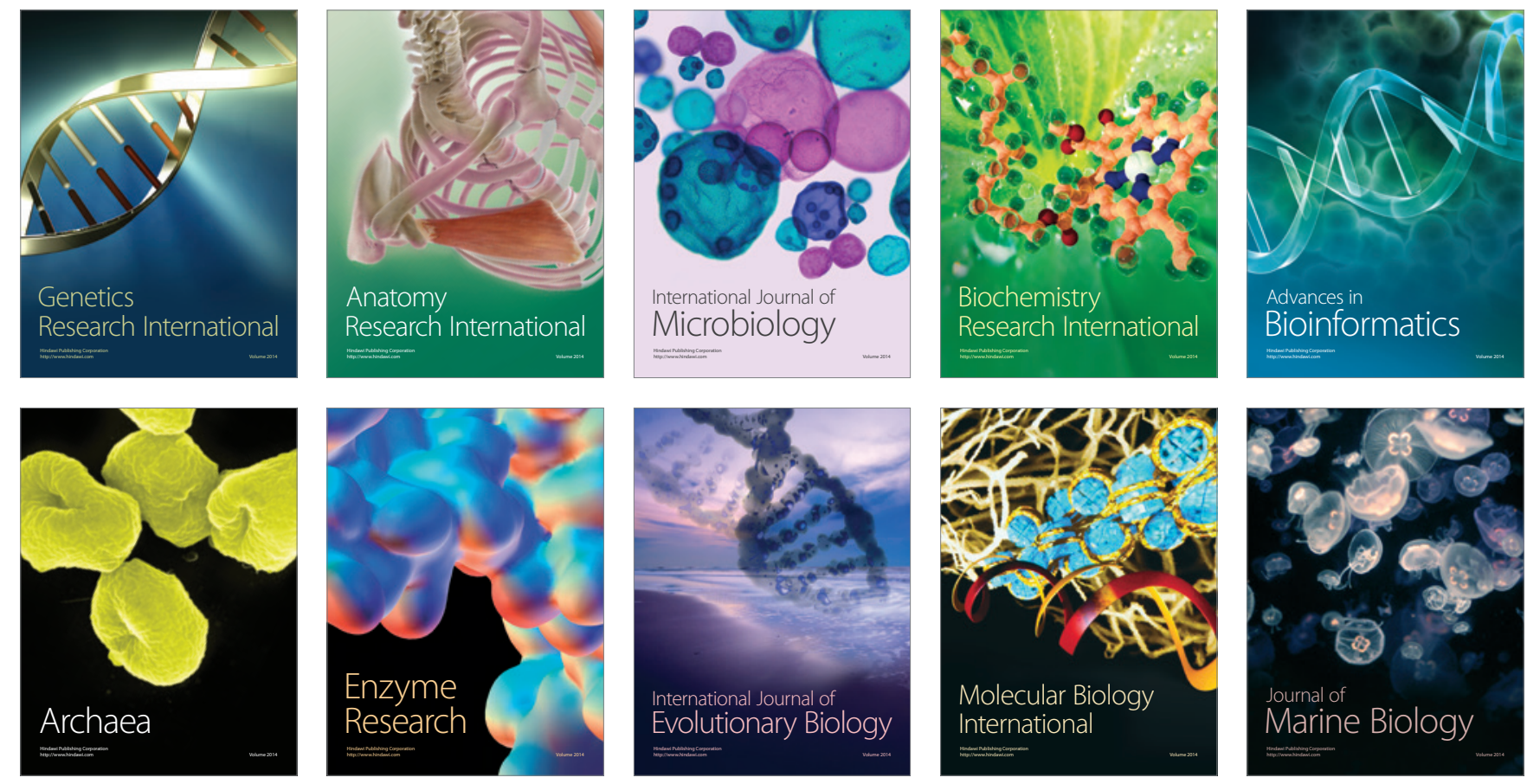